\title{
Artroplastía total de rodilla bilateral en un tiempo quirúrgico asistida por computadora
}

\section{Computer-assisted total bilateral knee arthroplasty in one surgical time}

\author{
Romero-Medina A,* Ortiz-Aguilar LS, ${ }^{\ddagger}$ García-Linage R, ${ }^{\ddagger}$ Saucedo-Moreno EM ${ }^{\S}$ \\ Hospital Ángeles Mocel.
}

RESUMEN. Introducción: La artroplastía total de rodilla navegada utiliza un sistema guiado por computadora que proporciona información inmediata de condiciones transoperatorias precorte de la rodilla, con relación a la alineación del miembro pélvico. Material y métodos: Estudio observacional, descriptivo, efectuado de Marzo de 2003 a Febrero de 2019. Se realizó artroplastía total de rodilla bilateral navegada en un mismo tiempo quirúrgico por un cirujano, evaluando función y dolor según las escalas WOMAC, EVA y arcos de movilidad de ambas rodillas. Se estudiaron dos grupos de pacientes: el primero representa prequirúrgicos y el segundo postquirúrgicos. Se aplicó prueba t de Student y $\chi^{2}$ para el análisis estadístico. Resultados: Se estudiaron 31 pacientes (62 prótesis), 83.9\% del sexo femenino y $16.1 \%$ del masculino, edad media 67.32 años, media de seguimiento 6.55 años $( \pm 3.8)$. Se identificó que $100 \%$ de los pacientes intervenidos en ambas rodillas tienen una desviación entre $0^{\circ}$ y $2^{\circ}$ medida en el eje mecánico. La escala WOMAC mostró una media de $22.71 \pm 3.34$ prequirúrgica y $4.16 \pm 1.84$ postquirúrgica, con diferencias estadísticamente significativas. La escala visual análoga media fue de $9.06 \pm$ 0.814 prequirúrgico y $2.35 \pm 1.427$ postquirúrgico. Conclusiones: Esta técnica es confiable, segura y satisfactoria. Se evidenciaron excelentes resultados clínicos y radiográficos respecto al posicionamiento de los componentes protésicos.

Palabras clave: ATR, bilateral, navegación, resultados, cirugía.
ABSTRACT. Introduction: Total navigated knee replacement uses a computer-guided system, which provides immediate information on pre-cut transoperative conditions of the knee, in relation to pelvic limb alignment. Material and methods: Observational, descriptive study conducted from March 2003 to February 2019. Total bilateral knee replacement was performed at the same time surgically by a surgeon, evaluating function and pain on the WOMAC, EVA, and range of motion scores of both knees. Two groups of patients were studied: the first represents presurgical and the second postsurgical. Student's t-test and $\chi^{2}$ were applied for statistical analysis. Results: 31 patients (62 prostheses), 83.9\% of the female sex and $16.1 \%$ male, average age 67.32 years, average follow-up 6.55 years $( \pm 3.8)$ were studied. It was identified that $100 \%$ of the patients in both knees have a deviation between $0^{\circ}$ and $2^{\circ}$ measured in the mechanical axis. The WOMAC scale showed an average of 22.71 \pm 3.34 presurgical and $4.16 \pm 1.84$ ) post-surgical, with statistically significant differences. The average analog visual scale was $9.06 \pm 0.814$ presurgical and $2.35 \pm 1.427$ post-surgical. Conclusions: This technique is reliable, safe and satisfactory. Excellent clinical and radiographic results were evident regarding the positioning of prosthetic components.

Keywords: TKR, bilateral, navigation, results, surgery.

\section{Nivel de evidencia: IV}

* Titular de la clínica de rodilla y hombro del Centro de Cirugía Ortopédica de Alta Especialidad Hospital Ángeles MOCEL.

* Residente de Ortopedia y Traumatología del Hospital Ángeles MOCEL avalada por la Facultad Mexicana de Medicina de la Universidad La Salle.

§ Servicio de Cirugía General, Hospital Ángeles MOCEL.

\section{Correspondencia:}

Dr. Alejandro Romero-Medina

General Juan Cano Núm. 107, San Miguel Chapultepec I Sección, Alcaldía Miguel Hidalgo, C.P. 11850, Ciudad de México.

E-mail: aromeromedina@yahoo.com

Recibido: 22-04-2020. Aceptado: 18-12-2020. 


\section{Introducción}

La artroplastía total de rodilla navegada (ATRN) es una técnica quirúrgica que se realiza cada vez con mayor frecuencia en diversos países y se lleva a cabo mediante un sistema guiado por computadora, la cual proporciona información en tiempo real de las condiciones transoperatorias precorte de la rodilla con relación a la alineación del miembro pélvico, profundidad de las lesiones condrales, estabilidad capsuloligamentaria y movilidad, guiando e informando al cirujano secuencialmente en la toma de datos para realizar con precisión milimétrica los cortes óseos y el balance ligamentario dinámico durante toda la movilidad articular de la rodilla para una adecuada alineación y estabilidad articular en extensión. Además, documenta la condición previa y final del procedimiento. . $^{1,2,3,4}$

El objetivo de este trabajo es evaluar el resultado a mediano y largo plazo en relación al dolor, movilidad, función y la satisfacción de los pacientes sometidos a esta técnica, además de describir las complicaciones inherentes a este procedimiento.

\section{Material y métodos}

Estudio observacional, descriptivo durante el período comprendido de Marzo de 2003 a Febrero de 2019. A los pacientes se les realizó artroplastía total de rodilla bilateral navegada en un mismo tiempo quirúrgico. Las prótesis utilizadas fueron Search Evolution y Columbus ${ }^{\circledR}$, (B-Braun Aesculap) y en todas las prótesis se empleó un navegador Ortho Pilot ${ }^{\circledR} .5$

Los criterios de inclusión fueron: pacientes mayores de 60 años y menores de 80 años de edad con diagnóstico de gonartrosis bilateral grado III y IV de acuerdo a la clasificación radiológica de Kellgren-Lawrence ${ }^{6}$ Los criterios de exclusión fueron los siguientes: riesgo prequirúrgico ASA III, obesidad mórbida, comorbilidades descontroladas, antecedente de enfermedades o alteraciones neurológicas o musculoesqueléticas que puedan afectar la capacidad de marcha, antecedentes quirúrgicos previos de osteotomías alineadoras, osteosíntesis en fémur y/o tibia, artrodesis de alguna articulación de miembro pélvico, artroplastía de rodilla con medicamentos administrados durante el año previo a la cirugía y que dicho tratamiento médico pudiera interferir con el metabolismo óseo o la cicatrización.

Los instrumentos de evaluación de función y dolor fueron las escalas Western Ontario and McMaster Universities Arthritis Index (WOMAC) 7 que evalúa dolor, rigidez y capacidad funcional de cada paciente, la escala visual análoga del dolor (EVA) y la movilidad articular de ambas rodillas. La evaluación postoperatoria se hizo a los seis meses y consecutivamente cada año hasta el cierre de este informe. El seguimiento mínimo fue de un año y máximo de 15 años.

Registramos los minutos del tiempo quirúrgico y los mililitros del sangrado transquirúrgico, obteniendo los datos de la hoja de registro de anestesiología.
Los pacientes fueron operados por el mismo cirujano. La primera rodilla en abordarse fue la más dañada. A todos se les realizó la misma técnica quirúrgica con un abordaje convencional, vaciamiento sanguíneo con venda Smarch e isquemia a $250 \mathrm{mmHg}$, resección parcial de Hoffa y membrana sinovial del compartimento anterior femoral. Colocación de diodos receptores y espejo de infrarrojo, emitidos por la cámara del navegador, uno en fémur y otro en tibia. El navegador obtiene los puntos espaciales de la rodilla marcados por el cirujano mediante la utilización de un puntero, captado por una emisora de infrarrojos de doble cabezal en el navegador, logrando la integración de datos en la computadora como tamaño, forma, volumen, profundidad de los defectos, alineación del miembro pélvico, centros kinemáticos de cadera, rodilla, tobillo y brecha de flexoextensión. ${ }^{8}$ Concluida la toma de datos y con esta información integrada en el navegador, se inicia el procedimiento de forma secuencial con el corte de la meseta tibial perpendicular a su eje y enseguida el balance de los ligamentos colaterales en flexoextensión. Con este corte inicial y la brecha de flexoextensión se hace la planeación transoperatoria del corte femoral, determinando junto con el navegador el

Grado de alineación eje mecánico izquierdo

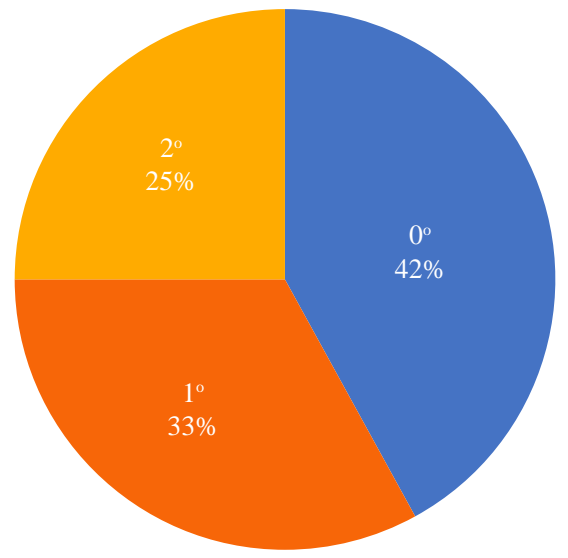

Grado de alineación eje mecánico derecho

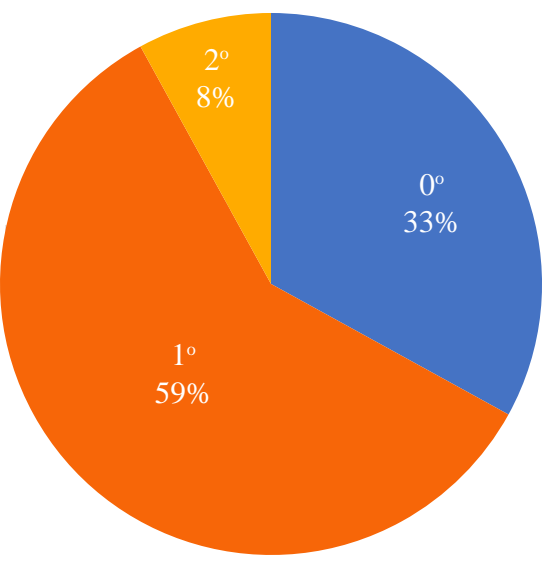

Figura 1: Alineación postoperatoria por lado operado. 
Tabla 1: Resultados entre grupo prequirúrgico y postquirúrgico.

\begin{tabular}{|c|c|c|c|c|}
\hline & Grupo 1 & Grupo 2 & & \\
\hline & Preqx & Postqx & $\mathrm{p}$ & IC 95\% \\
\hline WOMAC & $22.71 \pm 3.34$ & $4.16 \pm 1.84$ & 0.000 & 17.16 a 19.92 \\
\hline EVA & $9.06 \pm 0.814$ & $2.35 \pm 1.427$ & 0.000 & 6.140 а 7.279 \\
\hline Flexión derecha & $122.68 \pm 7.20$ & $127.10 \pm 4.23$ & 0.005 & -7.43 a -1.410 \\
\hline Flexión izquierda & $121.10 \pm 7.52$ & $126.90 \pm 4.527$ & 0.000 & -8.96 a 1.265 \\
\hline Extensión derecha & $3.35 \pm 1.74$ & $2.16 \pm 0.96$ & 0.001 & -0.477 a 1.910 \\
\hline Extensión izquierda & $2.81 \pm 1.53$ & $1.84 \pm 0.96$ & 0.004 & 0.315 a 1.620 \\
\hline
\end{tabular}
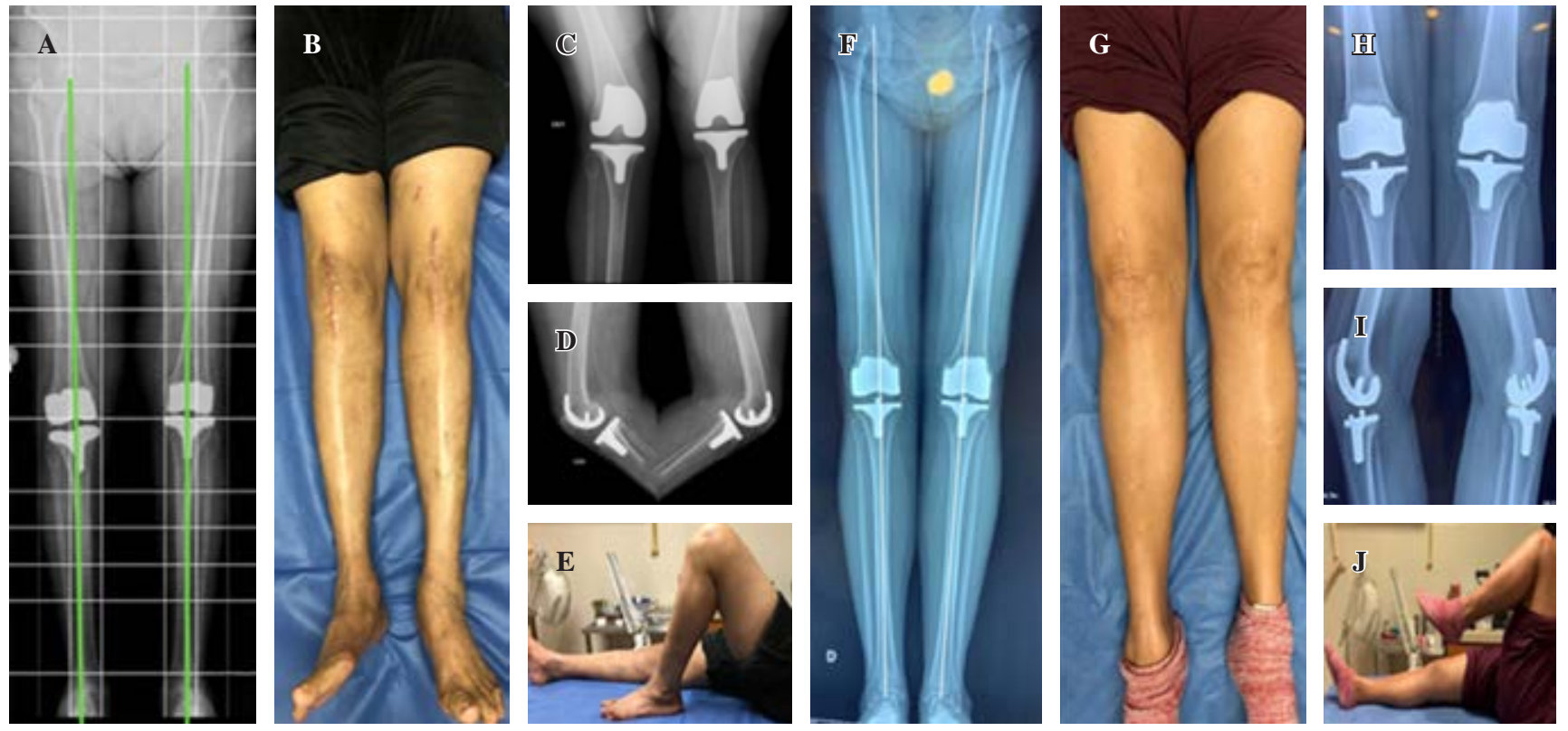

Figura 2: De A) a E): eje mecánico, resultado clínico ap, rx ap, rx lateral y resultado clínico lateral rodilla izquierda de la paciente \#12 a dos años postquirúrgicos. De F) a J): eje mecánico, resultado clínico ap, rx ap, rx lateral y resultado clínico lateral rodilla izquierda de la paciente \#13 a cinco años postquirúrgicos.

tamaño, rotación y colocación del componente femoral para lograr además el balance de ligamentos y eje mecánico de la extremidad. Con los cortes realizados se presentan los componentes de prueba y se verifica estabilidad, alineación y movilidad de la rodilla. El abordaje de la segunda rodilla se efectúa mientras se lavan y limpian fémur y tibia y se cementan los componentes de la prótesis de la primera rodilla con el objetivo de lograr disminuir los tiempos muertos transquirúrgicos. A ningún paciente se le colocó componente patelar, únicamente se realizó superficialización regularizando la superficie y retirando osteofitos, además de denervación periférica.

El registro de las variables de cada paciente se hizo en un programa Excel de Microsoft Windows ${ }^{\circledR}$, el análisis estadístico se realizó con el software SSPS versión $20.0^{\circledR}$ (SPSS, Chicago EUA). Para llevar a cabo el estudio utilizamos dos grupos: el grupo 1 representa los datos en el preoperatorio y el grupo 2 los datos en el postoperatorio. Aplicamos una prueba t de Student para datos emparejados y se expresan como media \pm desviación estándar (DE), mientras que las variables categóricas fueron analizadas con $\chi^{2}$. Se utilizó el valor menor de 0.05 para una p significativa con IC de $95 \%{ }^{9}$

\section{Resultados}

Estudiamos un total de 31 pacientes, de los cuales 83.9\% corresponde al sexo femenino (26) y $16.1 \%$ al masculino (5). Con una edad media de $67.32 \pm 7.64$ años y una media en años de seguimiento de $6.55( \pm 3.8)$. Se colocaron 24 prótesis Search Evolution ${ }^{\circledR}$ (de 2003 a 2012) y 38 prótesis Columbus $^{\circledR}$ (de 2012 a 2019).

En relación al eje mecánico, 100\% de los pacientes intervenidos tienen una desviación entre $0^{\circ}$ y $2^{\circ}$ en el plano coronal (Figura 1). La función con los resultados de la escala de WOMAC prequirúrgica y postquirúrgica mostró una media de $22.71 \pm 3.34$ prequirúrgica y $4.16 \pm 1.84$ postquirúrgica, 
con diferencias estadísticamente significativas $\mathrm{p}=0.000$ (IC 95\% de 17.16 a 19.92) (Tabla 1).

La evolución del dolor según la escala visual análoga del dolor (EVA), los resultados prequirúrgicos y postquirúrgicos fueron los siguientes: EVA media de $9.06 \pm 0.814$ para el prequirúrgico, en comparación con $2.35 \pm 1.427$ para el grupo postquirúrgico, con diferencias significativas $\mathrm{p}=$ 0.000 (IC 95\% de 6.140 a 7.27) (Tabla 1).

En relación a la movilidad articular de flexoextensión bilateral obtenemos mejoría con diferencias clínicas significativas tanto derecha como izquierda con $\mathrm{p}<0.05$ (Tabla 1). Se muestra resultado clínico y eje mecánico radiográfico de cuatro pacientes a diferentes años postquirúrgicos (Figuras 2 y3).

Analizamos también el tiempo quirúrgico del procedimiento bilateral y el sangrado transquirúrgico y postquirúrgico. Respecto al tiempo, obtuvimos una media de 212.08 minutos ( \pm 52.08). Y del sangrado transquirúrgico y postquirúrgico de ambas rodillas obtuvimos una media de $504 \mathrm{ml}( \pm 396)$.

Las complicaciones se presentaron en dos pacientes con dehiscencia superficial de la herida menor de $2 \mathrm{~cm}$ (sólo piel), resuelta con la aplicación de vendoletes. El resto de las complicaciones asociadas con mayor frecuencia a la ATR fueron nulas en nuestros pacientes, tales como trombosis venosa, tromboembolia pulmonar, infección y/o aflojamiento periprotésico.

\section{Discusión}

El éxito de la artroplastía total de rodilla navegada (ATRN) radica en la selección apropiada del paciente, el diseño de la prótesis, la selección del tamaño ideal del componente protésico y la colocación precisa de los mismos con el objetivo de lograr la correcta alineación del miembro pélvico y consecuentemente evitar desgaste temprano de los implantes, aflojamiento protésico y por último el fracaso en el tratamiento. ${ }^{8,10,11,12,13}$

En la literatura médica la información referente a los resultados funcionales y a las complicaciones de esta técnica quirúrgica es escasa. ${ }^{14}$ Este artículo original demuestra buenos resultados basándose en la escala de WOMAC, además reporta una incidencia de cero en las complicaciones de la ATR bilateral simultánea asistida por navegación; sin embargo, reconocemos el tamaño reducido de la muestra a lo largo de 16 años de estudio.

El resultado del eje mecánico obtenido en nuestros pacientes coincide con los resultados de varios metaanálisis, ${ }^{14}$ proponiendo esta técnica de elección en deformidades en varo o valgo mayores de $10^{\circ}$ para aumentar la precisión del eje mecánico final y el posicionamiento de los componentes protésicos de tibia y fémur. ${ }^{15,16}$ En nuestro estudio no incluimos los valores preoperatorios del eje mecánico para hacer una comparación objetiva, sólo pudimos comprobar que al utilizar esta técnica existen mejorías significativas tanto en funcionalidad, dolor y alineación del eje mecánico a mediano y largo plazo. Consideramos la necesidad de seguir a los pacientes por un período más largo para poder emitir resultados a largo plazo. ${ }^{14}$ Logramos una media en años de seguimiento de 6.55 con buenos resultados funcionales.

Ninguno de los pacientes presentó complicaciones inmediatas asociadas a la ATR como tromboembolia pulmonar, flebitis, trombosis venosa, infección periprotésica o aflojamiento de los componentes protésicos. Destacamos el apego a protocolos preoperatorios para que las condiciones y seguridad de los pacientes permitan realizar el procedimiento quirúrgico sin riesgos mayores, ${ }^{17,18,19,20,21}$ aunque la ausencia
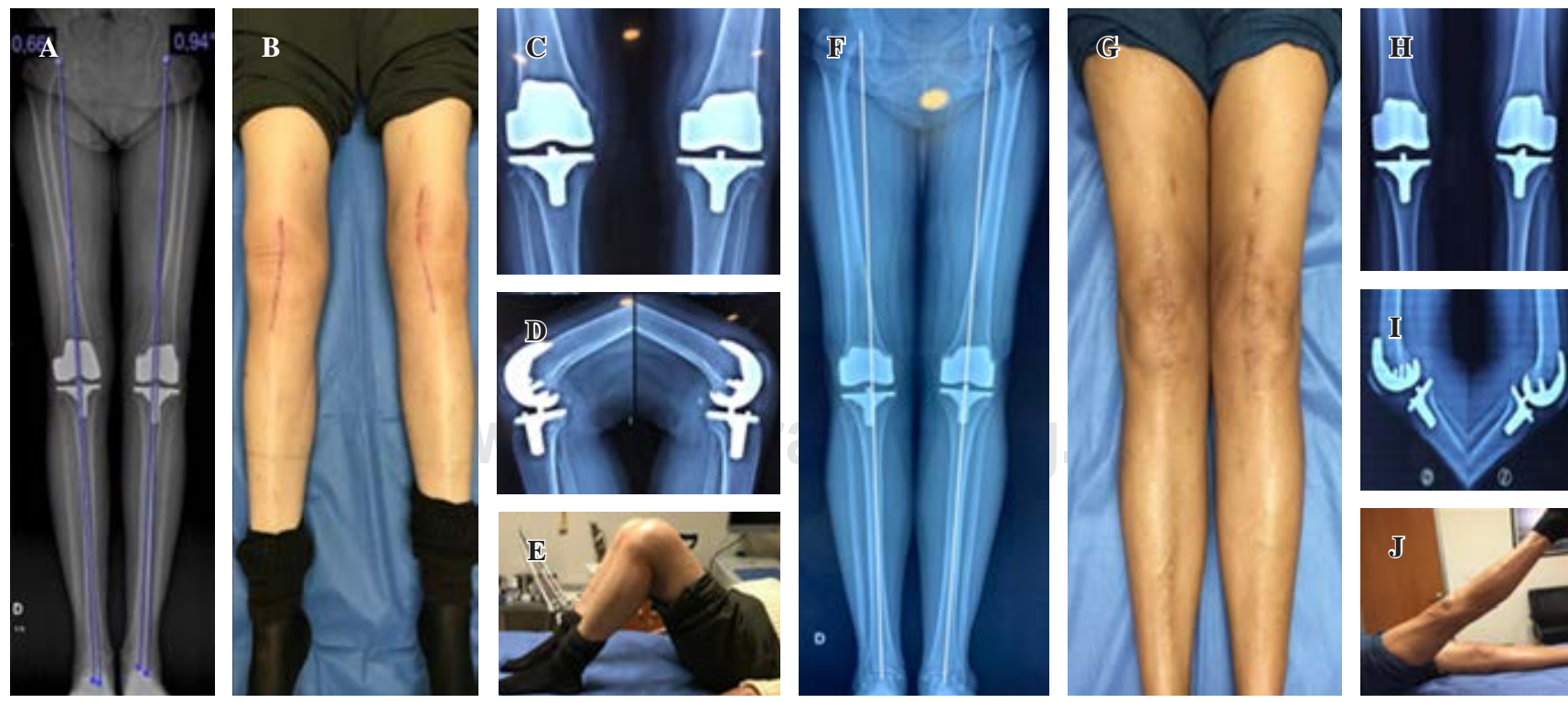

Figura 3: De A) a E) : eje mecánico, resultado clínico ap, rx ap, rx lateral y resultado clínico lateral ambas rodillas de la paciente \#9 a tres años postquirúrgicos. De F) a J): eje mecánico, resultado clínico ap, rx ap, rx lateral y resultado clínico lateral rodilla derecha de la paciente \#23 a ocho años postquirúrgicos. 
de un grupo control no nos permite más que comentarios subjetivos. El tiempo quirúrgico no fue mayor que el reportado en estudios donde se realiza ATR bilateral comparando técnicas asistidas por computadora contra técnicas convencionales en un solo tiempo quirúrgico.

Yongjian Qi y colaboradores no identifican en su estudio un incremento en las complicaciones estadísticamente significativas, estudio que reporta tasa de $9.3 \%$ en ATR bilateral convencional (no navegada), vs. 8.6\% en la ATR unilateral convencional. ${ }^{22}$ Sin embargo, se ha identificado que las complicaciones cardiovasculares se incrementan en personas mayores de 80 años que son sometidas a ATR bilateral convencional. ${ }^{23}$ Es importante tener en cuenta que una contraindicación para la realización de ATR bilateral en nuestra serie fue la existencia de alguna patología cardiovascular. ${ }^{24,25}$

Dentro de las limitantes de nuestro estudio hay que considerar que los procedimientos se llevaron a cabo en un centro quirúrgico privado y que el tamaño de muestra fue relativamente pequeño. A pesar de la desventaja del tamaño reducido de la muestra y de haberse realizado el estudio en un solo hospital, es relevante que nuestra incidencia de complicaciones asociadas a ATR es nula, comparándonos con los estudios de ATR bilateral convencional. No encontramos en la literatura ningún estudio similar de prótesis navegada bilateral, por lo que no hemos podido comparar nuestros resultados obtenidos con esta técnica.

\section{Conclusiones}

Obtuvimos mejoría significativa tanto en función, dolor $\mathrm{y}$ arco de movimiento postoperatoria. Es una técnica confiable, segura y satisfactoria para el tratamiento de pacientes con gonartrosis bilateral grado III y IV.

Referencias

1. Spencer JM, Chauhan SK, Sloan K, Taylor A, Beaver RJ. Computer navigation versus conventional total knee replacement. No difference in functional results at two years. J Bone Joint Surg Br. 2007; 89-B(4): 477-80.

2. Inui H, Taketomi S, Nakamura K, Takei S, Takeda H, Tanaka S, et al. Influence of navigation system updates on total knee arthroplasty. BMC Sports Sci, Med Rehabil. 2013, 5: 10.

3. Zanasi S. Innovations in total knee replacement: new trends in operative treatment and changes in perioperative management. Eur Orthop Traumatol. 2011; 2: 21-31.

4. Manfrin FG, Maestu R. Artroplastía total de rodilla con asistencia de navegación. Nuestra experiencia. Rev Asoc Argent Ortop Traumatol. 2010; 74: 341-6.

5. Stulberg SD, Picard F, Saragaglia D. Computer-assisted total knee replacement arthroplasty. Operative Techniques in Orthopaedics. 2000; (10): 25-39.
6. Rick W Wright1, MARS Group. Osteoarthritis classification scales: interobserver reliability and arthroscopic correlation. $J$ Bone Joint Surg Am. 2014; 96(14): 1145-51.

7. Escobar A, Vrotsou K, Bilbao A, Quintana JMA, García PL, HerreraEspiñeira C. Validación de una escala reducida de capacidad funcional del cuestionario WOMAC. Gac Sanit. 2011; 25(6): 513-8.

8. Siston RA, Giori NJ, Goodman SB, Delp SL. Surgical navigation for total knee arthroplasty: a perspective. J Biomech. 2007; 40(4): 728-35.

9. Saucedo-Moreno EM, Rodríguez F. Estadística en cirugía, como entender y aplicar conceptos básicos. Cir Cir. 2019; 87: 692-7.

10. Saragaglia D, Picard F. Computer-assisted implantation of total knee endoprothesis with no peroperative imagin: the kinematic model. Navigation and robotics in total joint and spine surgery. SpringerVerlag Berlin Heidelberg. 2004; 32: 226-233.

11. Ochoa R. Cuadra Castillo M. Artroplastía total de rodilla navegada. ¿Por qué utilizarla? Acta Ortop Mex. 2013; 27(3): 205-10.

12. Confalonieri N, Biazzo A. Computer-assisted surgery in total knee replacement: advantages, surgical procedure and review of the literature. Acta Biomed. 2019; 90(1): 16-23.

13. Ren $\mathrm{Y}, \mathrm{Cao} \mathrm{S}, \mathrm{Wu}$ J, Weng X, Feng B. Efficacy and reliability of active robotic-assisted total knee arthroplasty compared with conventional total knee arthroplasty: a systematic review and metaanalysis. Postgrad Med J. 2019; 95(1121): 125-33.

14. Hetaimish BM, Moin Khan M, Simunovic N, Al-Harbi HH, Bhandari M, Zalzal PK. Meta-analysis of navigation vs conventional total knee arthroplasty. J Arthroplasty. 2012; 27(6): 1177-82.

15. Hernández-Vaquero D, Noriega-Fernandez A, Roncero-Gonzalez S, Perez-Coto I, Sierra-Pereira AA, Sandoval-Garcia MA. Agreement in component size between preoperative measurement, navigation and final implant in total knee replacement. J Orthop Translat. 2018; 18: 84-91.

16. Hernández-Vaquero D, Suarez-Vazquez A, Sandoval-Garcia MA, Noriega-Fernandez A. Computer assistance increases precision of component placement in total knee arthroplasty with articular deformity. Clin Orthop Relat Res. 2010; 468: 1237-41.

17. Kurtz SM, Lau E, Schmier J, Ong KL, Zhao K, Parvizi J. Infection burden for hip and knee arthroplasty in the United States. $J$ Arthroplasty. 2008; 23(7): 984-91.

18. Pulido L, Ghanem E, Joshi A, Purtill JJ, Parvizi J. Periprosthetic joint infection: the incidence, timing and predisposing factors. Clin Orthop Relat Res. 2008; 466: 1710-5.

19. Vélez-de Lachica JC, Santos-Briones JI, Inzunza-Sánchez JM. Aplicación profiláctica de sulfato de calcio medicado en prótesis total de cadera no cementada. Acta Ortop Mex. 2019; 33(2): 67-72.

20. Urban MK. Anestesia para la cirugía ortopédica. En: Miller RD. editors. Miller's anesthesia. Philadelphia: Elsevier Churchill Livingstone, 2010.

21. Bierbaum B, Callaghan JJ, Galante JO, Rubash HE, Tooms RE, Welch RB. An analysis of blood management in patients having a total hip or knee arthroplasty. J Bone Joint Surg Am. 1999; 81(1): 2-10.

22. Qi Y, Tie K, Wang H, Pan Z, Zhao X, Chen H, et al. Perioperative comparison of blood loss and complications between simultaneous bilateral and unilateral total knee arthroplasty for knee osteoarthritis. Knee. 2017; 24(6): 1422-7.

23. Lynch NM, Trousdale RT, Ilstrup DM. Complications after concomitant bilateral total knee arthroplasty in elderly patients. Mayo Clin Proc. 1997; 72: 799-805.

24. Yoon HS, Han CD, Yang IH Comparison of simultaneous bilateral and staged bilateral total knee arthroplasty in terms of perioperative complications. J Arthroplasty. 2010; 25(2): 179-85.

25. Lane GJ, Hozack WJ, Shah S, Rothman RH, Booth RE Jr, Eng $\mathrm{K}$, et al. Simultaneous bilateral versus unilateral total knee arthroplasty. Outcomes analysis. Clin Orthop Relat Res. 1997; (345): 106-12. 